\title{
DEFLECTIONS OF FAST CORONAL MASS EJECTIONS AND THE PROPERTIES OF ASSOCIATED SOLAR ENERGETIC PARTICLE EVENTS
}

\author{
S. W. KahleR ${ }^{1}$, S. AKiyAma ${ }^{2}$, And N. Gopalswamy ${ }^{3}$ \\ ${ }^{1}$ Air Force Research Laboratory, Space Vehicles Directorate, 3550 Aberdeen Avenue, Kirtland AFB, NM 87117, USA; AFRL.RVB.PA@kirtland.af.mil \\ ${ }^{2}$ Institute for Astrophyics and Computational Sciences, The Catholic University of America, Washington, DC 20064, USA \\ ${ }^{3}$ NASA Goddard Space Flight Center, Greenbelt, MD 20771, USA \\ Received 2012 March 30; accepted 2012 May 27; published 2012 July 12
}

\begin{abstract}
The onset times and peak intensities of solar energetic particle (SEP) events at Earth have long been thought to be influenced by the open magnetic fields of coronal holes $(\mathrm{CHs})$. The original idea was that a $\mathrm{CH}$ lying between the solar SEP source region and the magnetic footpoint of the $1 \mathrm{AU}$ observer would result in a delay in onset and/or a decrease in the peak intensity of that SEP event. Recently, Gopalswamy et al. showed that CHs near coronal mass ejection (CME) source regions can deflect fast CMEs from their expected trajectories in space, explaining the appearance of driverless shocks at $1 \mathrm{AU}$ from CMEs ejected near solar central meridian (CM). This suggests that SEP events originating in CME-driven shocks may show variations attributable to $\mathrm{CH}$ deflections of the $\mathrm{CME}$ trajectories. Here, we use a $\mathrm{CH}$ magnetic force parameter to examine possible effects of $\mathrm{CHs}$ on the timing and intensities of 41 observed gradual $E \sim 20 \mathrm{MeV}$ SEP events with CME source regions within $20^{\circ}$ of $\mathrm{CM}$. We find no systematic $\mathrm{CH}$ effects on SEP event intensity profiles. Furthermore, we find no correlation between the CME leading-edge measured position angles and SEP event properties, suggesting that the widths of CME-driven shock sources of the SEPs are much larger than the CMEs. Independently of the SEP event properties, we do find evidence for significant CME deflections by $\mathrm{CH}$ fields in these events.
\end{abstract}

Key words: acceleration of particles - Sun: coronal mass ejections (CMEs) - Sun: flares - Sun: particle emission

Online-only material: color figures

\section{INTRODUCTION}

\subsection{SEP Events and Coronal Holes}

Despite their important space weather effects (Bothmer \& Zhukov 2007; Guetersloh \& Zapp 2010), the occurrence and timing of gradual solar energetic $(E>10 \mathrm{MeV})$ particle (SEP) events are difficult to predict (Crosby 2007; Kahler et al. 2007). The consensus view is that the SEPs are produced by shock acceleration ahead of fast coronal mass ejections (CMEs; Reames 1999; Cliver 2009). However, the intensities and timescales of the SEP events show large variations even within limited ranges of solar longitude source regions and CME speeds and widths (Gopalswamy et al. 2004; Kahler 2005).

The SEP event profiles observed at $1 \mathrm{AU}$ depend on a number of unknown and often unobservable factors, such as the detailed interaction between the CME-driven shock and the SEP seedparticle population (Tylka \& Lee 2006; Klecker et al. 2007). A relatively unexplored area is the role of the coronal environment into which the fast CME propagates. Gopalswamy et al. (2002, 2004) found that SEP event peak intensities were substantially enhanced when the shock-driving CME was preceded within $24 \mathrm{hr}$ by another CME of width $>60^{\circ}$ from the same or a nearby solar source. Their result was confirmed by Kahler \& Vourlidas (2005), who also found that CMEs producing more intense SEP events were more massive and more likely to be streamer-blowout CMEs. The reasons for these differences are not clear.

Observations of SEP events within various solar wind (SW) structures at $1 \mathrm{AU}$ give little indication of the importance of those structures for SEP events. The frequency and sizes of SEP events (Kahler 2004) as well as their timescales (Kahler 2005) in fast SW streams are comparable to those in slow SW streams. More recently, the SEP elemental composition observed at 5-10 MeV nucleon $^{-1}$ has been found not to differ between the two kinds of streams (Kahler et al. 2009). SEPs can also propagate to very high $\left(>70^{\circ}\right)$ latitudes (Sanderson 2004; Malandraki et al. 2009) and result in impressively spatially uniform distributions across broad $\left(>160^{\circ}\right)$ longitude ranges during event decay phases (McKibben et al. 2003; Reames et al. 1997).

Since the SW high-speed streams originate from coronal holes (CHs; Wang 2009), these results suggest that CHs are not a significant factor in the properties of SEP events observed at $1 \mathrm{AU}$. Contrary to this expectation, however, Kunches \& Zwickl (1999) found that the times from flare X-ray maxima to 1 AU onsets of $>25 \mathrm{MeV}$ proton events during 1976-1997 were typically longer by periods of hours when a $\mathrm{CH}$ lay between the $\mathrm{CME}$ source region and the $1 \mathrm{AU}$ magnetic footpoint. Their result was perhaps inconsistent with later studies of front-side, west-hemisphere fast halo CMEs by Shen et al. (2006, 2010). For each CME they looked for an associated $1 \mathrm{AU} E>10 \mathrm{MeV}$ or $>50 \mathrm{MeV}$ SEP event and for the area, shortest distance, and relative location of any large $\mathrm{CH}$ near the $\mathrm{CME}$ source region. They found that the $\mathrm{CH}$ proximities, areas, and locations relative to the CME source and Earth magnetic connection were not factors in the occurrence of associated SEP events. Shen et al. (2006, 2010) considered whether SEP events occurred for given fast halo CMEs while Kunches \& Zwickl (1999) compared onset intervals for given SEP events, so their results are not directly comparable. However, the two works suggest opposite answers to the basic question of whether $\mathrm{CHs}$ play any role in the properties of SEP events, and the question remains controversial.

How can a $\mathrm{CH}$, or its high-speed stream, be a factor in the characteristics of an SEP event? The implication in the Kunches \& Zwickl (1999) study was that either the SEPs must propagate from the shock source across the intermediate $\mathrm{CH}$ field lines to 


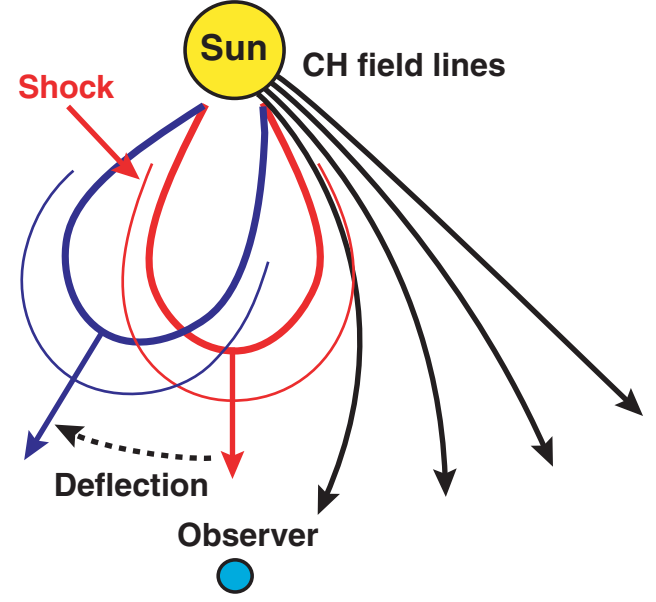

Figure 1. Schematic showing how the pressure of magnetic fields might deflect the propagation direction of a CME. The CME and preceding shock (red lines) that would have propagated toward the observer (blue dot) are deflected eastward (blue lines) by the fields extending from the $\mathrm{CH}$ lying west of the CME. Adapted from Gopalswamy et al. (2010).

(A color version of this figure is available in the online journal.)

reach those connecting to Earth or the speed or strength of the shock producing the SEPs is diminished as it crosses the field lines extending from the $\mathrm{CH}$. The latter possibility of diminished SEP production in weaker shocks traversing the higher-Alfvén speed regions characteristic of the $\mathrm{CH}$ (Kahler \& Reames 2003) also applies to coronal field regions adjacent to the CME.

\subsection{CH Deflections of CMEs}

Here we explore an alternative possibility that the $\mathrm{CH}$ fields affect coronal/interplanetary SEP production by latitudinally and/or longitudinally deflecting the CME and its preceding shock envelope. This work is motivated by the recent discovery (Gopalswamy et al. 2009) that some disk-center CMEs produced shocks at $1 \mathrm{AU}$ without accompanying in situ observations of the associated interplanetary CME (ICME) drivers. Most such driverless shocks occur only from CMEs near the solar limbs, but these disk-center CMEs were located adjacent to CHs which deflected the CMEs away from the Sun-Earth line, by angles as large as $\sim 50^{\circ}$ (Gopalswamy et al. 2009, 2010). The $\mathrm{CH}$ deflection effect was quantified by a $\mathrm{CH}$ influence parameter (CHIP), which showed statistically that disk-center CMEs producing shocks with observed drivers were negligibly influenced by CHs. An extended statistical comparison (Mohamed et al. 2012) of disk-center CMEs producing the six driverless shocks, shocks with observed magnetic clouds, and shocks with ICMEs found the CHIPs to increase in the order of magnetic cloud, ICME, and driverless shocks. This result suggested magnetic flux ropes at the origin of all the disk-center CMEs, observable only when $\mathrm{CH}$ deflections are small.

Recent observations (Shen et al. 2011; Gui et al. 2011; Liu et al. 2010; Byrne et al. 2010; Lugaz et al. 2011) show that these CME deflections must happen within several $R_{\odot}$, where the shock is expected to be strongest. The magnetic connectivity of the SEP acceleration region to the observer may change from a favorable to an unfavorable one or vice versa, as indicated in the schematic of Figure 1. Displacements of SEP acceleration regions suggest a new SEP environmental factor worthy of further exploration. Latitudinal deflections of CMEs up to $\sim 40^{\circ}$, prominently toward the equator around solar minimum, have been studied with Solar and Heliospheric Observatory/Large
Angle and Spectrometric Coronagraph Experiment ( $\mathrm{SOHO} /$ LASCO; Gopalswamy \& Thompson 2000; Cremades et al. 2006; Wang et al. 2011), STEREO/SECCHI (Kilpua et al. 2009; Byrne et al. 2010; Shen et al. 2011; Zuccarello et al. 2012), and SMEI (Kahler \& Webb 2010) observations. The interpretation is that around solar minimum the CMEs are guided equatorward by strong polar $\mathrm{CH}$ magnetic fields. This effect is also obvious in the rolling motions of eruptive prominences erupting in the vicinity of CHs (Panasenco et al. 2011, 2012). Longitudinal CME deflections are also observed. A westward motion during the acceleration phase found in SECCHI CMEs by Liu et al. (2010) was attributed to coronal corotation of CME fields, although adjacent high-speed streams were invoked earlier by Wang et al. (2004) to explain an inferred systematic eastward deflection of fast CMEs. However, the physical reality of CME deflections by tens of degrees in both latitude and longitude is now well established by both models and observations.

Modeling of the magnetic-field environment has shown that CME deflections are consistent with the directions and magnitudes of the magnetic-field energy-density gradients (Shen et al. 2011; Gui et al. 2011). Furthermore, slow CMEs are deflected more than fast CMEs (Wang et al. 2004; Gui et al. 2011). An early version of a $\mathrm{CH}$ magnetic force vector $F$, derived from $\mathrm{CH}$ area and distance from the CME source and directed against CMEs, was consistent with observed latitudinal CME deflections (Cremades et al. 2006). Gopalswamy et al. (2009) modified $F$ to include the average $\mathrm{CH}$ magnetic field $B$ as a linear factor of their CHIP (Mohamed et al. 2012). Gopalswamy et al. (2010) then redefined $F$ to a pressure term with a $B^{2}$ factor, finding that $F$ was larger for the CMEs of driverless shocks than for the shocks with detected drivers at $1 \mathrm{AU}$.

The relevance of CME deflections to SEP events rests on two considerations. The shock flank on the $\mathrm{CH}$ side may be weakened because CHs generally have higher-Alfvén speeds as discussed above. The second is the question of shock connectivity to field lines well connected to 1 AU observers. A poorly connected (say from a site near the disk center) CME might become well connected if the CME nose is deflected to the west by a $\mathrm{CH}$ located between the disk center and the eruption site. On the other hand, the connectivity may be diminished if the $\mathrm{CH}$ is located to the west of the eruption region, as in Figure 1. The presence of one or more $\mathrm{CH}$ s near the $\mathrm{CME}$ source region could modify the SEP production in either case, and in this work we seek evidence for this effect.

\section{DATA ANALYSIS}

\subsection{Selection of SEP and CH Data}

For the analysis here, we use timing parameters and peak intensities of $20 \mathrm{MeV}$ SEP events measured with the EPACT instrument on the Wind spacecraft and associated with SOHO/LASCO CMEs. The event list of Table 1 of Kahler (2005) has been updated through 2008. Since any effects of $\mathrm{CHs}$ on CME and shock propagation and associated SEP acceleration should be most apparent during the rise phase of the SEP event, we select for analysis, besides the peak SEP intensity Ip, the SEP onset time $T_{O}$, defined as the time from CME onset to SEP onset at Wind, and $T_{R}$, the time from SEP onset to half-maximum of the SEP peak intensity (Figure 2). These SEP timescales can range up to an order of magnitude (Table 2, Kahler 2005), even within relatively narrow source longitude ranges. Here we look for any effects of $\mathrm{CHs}$ on the SEP event timescales and intensities. 


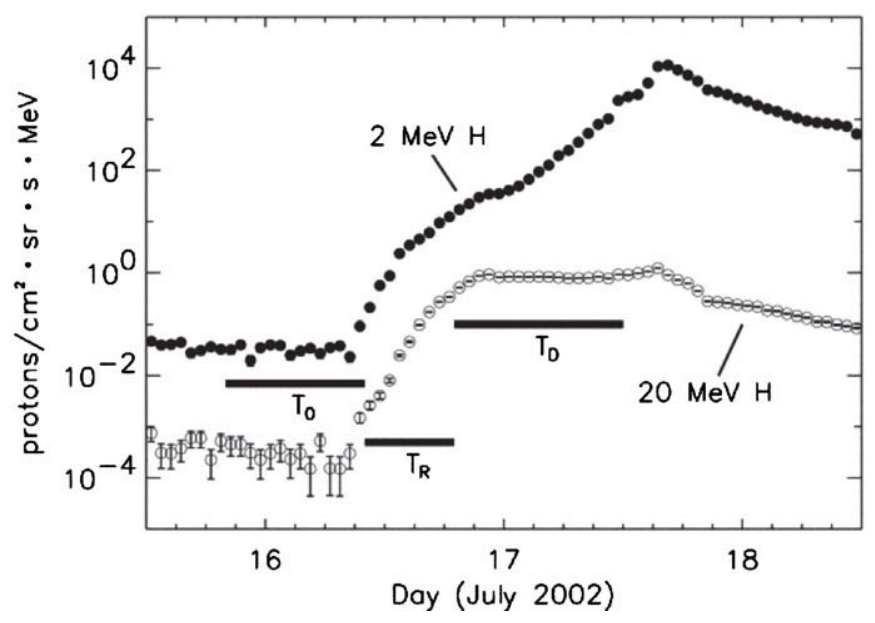

Figure 2. Plot of 2 and $20 \mathrm{MeV}$ proton intensities from the Wind/EPACT instrument during the SEP event of 2002 July 16 . The horizontal bars show $T_{O}$, the time from CME onset to SEP onset at Wind; $T_{R}$, the rise time from onset to half the peak intensity; and $T_{D}$, the event duration to half peak or shock. Only $T_{O}$ and $T_{R}$ are used in the analysis here.

Table 1

The CH $F$ Parameters for the 2004 November 7 SEP Event

\begin{tabular}{lcrrcccc}
\hline \hline Location & Area $^{\mathrm{a}}$ & Ave $B$ & Distance $^{\mathrm{b}}$ & $F$ & FPA $^{\mathrm{c}}$ & Flat & Flong \\
\hline S49W06 & $1.3 \mathrm{e}+11$ & 2.1 & $6.8 \mathrm{e}+05$ & $5.9 \mathrm{e}-01$ & $354^{\circ}$ & $5.6 \mathrm{e}-02$ & $5.9 \mathrm{e}-01$ \\
S05E11 & $7.9 \mathrm{e}+09$ & 3.6 & $3.9 \mathrm{e}+05$ & $1.9 \mathrm{e}-01$ & $298^{\circ}$ & $1.7 \mathrm{e}-01$ & $9.3 \mathrm{e}-02$ \\
N37E36 & $3.6 \mathrm{e}+10$ & -4.9 & $6.4 \mathrm{e}+05$ & $4.2 \mathrm{e}-01$ & $231^{\circ}$ & $3.3 \mathrm{e}-01$ & $-2.6 \mathrm{e}-01$ \\
N38E61 & $2.0 \mathrm{e}+10$ & -10.0 & $8.4 \mathrm{e}+05$ & $2.9 \mathrm{e}-01$ & $233^{\circ}$ & $2.3 \mathrm{e}-01$ & $-1.7 \mathrm{e}-01$ \\
N63E42 & $5.8 \mathrm{e}+10$ & -4.1 & $7.7 \mathrm{e}+05$ & $4.0 \mathrm{e}-01$ & $205^{\circ}$ & $1.7 \mathrm{e}-01$ & $-3.6 \mathrm{e}-01$ \\
Total $F$ & & & & $9.6 \mathrm{e}-01$ & $264^{\circ}$ & $9.6 \mathrm{e}-01$ & $-1.0 \mathrm{e}-01$
\end{tabular}

Notes.

a Area in units of $\mathrm{km}^{2}$

${ }^{\mathrm{b}}$ Distance in units of $\mathrm{km}$.

${ }^{\mathrm{c}} F$ position angle measured ccw from north.

CHs and CME source regions are best observed at solar central meridian $(\mathrm{CM})$, so we select a source longitude range of E20 $0^{\circ}$ to $\mathrm{W}^{\circ} 0^{\circ}$, which yields 41 SEP events near CM with relatively well-observed $\mathrm{CH}$ boundaries and intensities. We identify the $\mathrm{CME}$ source regions by the associated flare active region locations, and use the $S O H O$ EIT $284 \AA$ images to define the $\mathrm{CH}$ boundaries and the $\mathrm{SOHO}$ MDI images to determine the average $\mathrm{CH}$ magnetic fields. Following Gopalswamy et al. (2010), we calculate for each CME the influence parameter vector $F$, defined as

$$
F=B^{2} A / r^{2},
$$

where $A$ is the area of the $\mathrm{CH}, B$ is the average magnetic field in the $\mathrm{CH}$, and $r$ is the $\mathrm{CH}$ distance. $F$ for each $\mathrm{CH}$ is directed from the $\mathrm{CH}$ center to the $\mathrm{CME}$ source region, and the total $F$, in units of Gauss squared, is the vector sum of $F$ over all individual CHs. The position angle FPA is the assumed direction in which the $\mathrm{CH}$ fields are deflecting the CME, as indicated for an example $\mathrm{SEP}$ event in Figure 3. The parameters for each of the five $\mathrm{CHs}$ and their $F$ vectors in this case are given in Table 1, where the last two columns give the latitudinal Flat (positive is northward) and longitudinal Flong (positive is westward) vector components. The tail of the arrow showing $F$ is placed on the CME source region in Figure 3 to indicate the expected deflection direction of the CME.

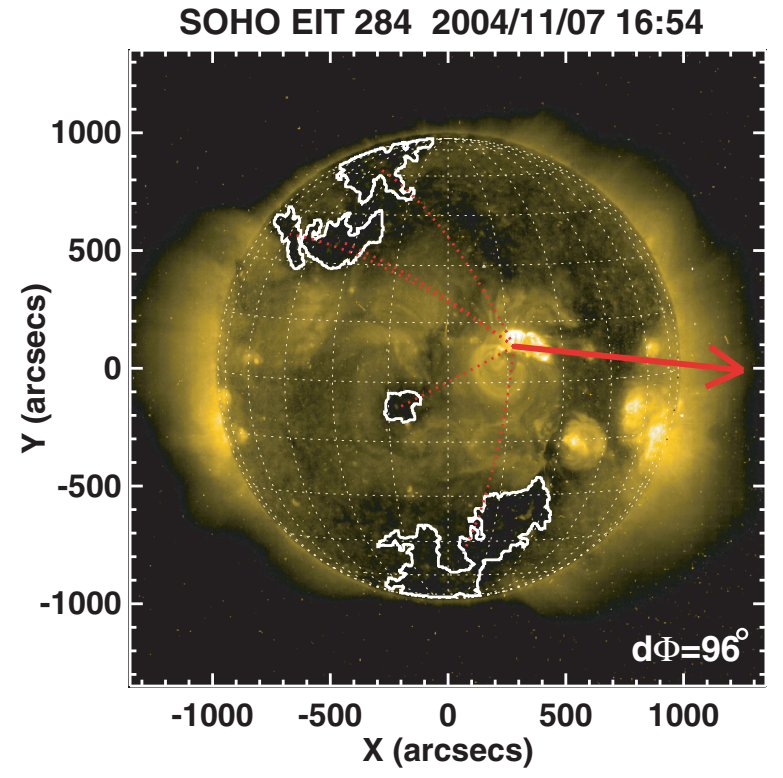

Figure 3. Full-disk EIT $284 \AA$ Amage on 2004 November 7 showing boundaries of the five $\mathrm{CHs}$ used to calculate the component $F$ vectors of Table 1 . The red dotted lines show the connecting distances $r$ and directions between the $\mathrm{CHs}$ and the $\mathrm{CME}$ source region for each component. The position angle of the summed $F$ vector (red arrow) is $264^{\circ}$ and is placed on the active region source of the CME at N09W17 to show the expected CME deflection direction.

(A color version of this figure is available in the online journal.)

Since the presumed influence of $\mathrm{CHs}$ on SEP events is produced by deflections of CMEs, we also look at the CME propagation directions given in the CDAW LASCO CME catalog. Of the $41 \mathrm{CMEs}, 30$ are full halos spanning $360^{\circ}$ of width, but each CME speed is determined from a leadingedge direction, the measurement position angle (MPA), selected from the first CME images. These directions are usually, but not always, near the central angle of the CME span. In our examination of the movies of subtracted C2 LASCO images, we found six cases of significant disagreements ranging from $25^{\circ}$ to $105^{\circ}$ between the catalog MPA and our determination of the primary CME PA. We used our 6 PAs and the catalog MPA values for the other $35 \mathrm{CMEs}$ in the data analyses.

\subsection{The Statistical Comparisons \\ 2.2.1. The CH Deflection Correlations}

In this statistical study, we look for correlations between pairs of SEP event and associated $\mathrm{CH} F$ parameters. The expectation is that CME deflections either westward or equatorward from highlatitude source regions will result in more favorable magnetic connections of shock accelerated SEPs to the field-line connections to the Earth, resulting in shorter SEP event timescales and/or higher peak intensities. For this purpose, we deal separately with the Flong and Flat components of $F$. We redefine Flat to be positive when pointing from the solar source region toward the ecliptic and negative when pointing poleward. The sign of Flat, i.e., whether the CME was being deflected equatorward or poleward, was determined after first calculating the solar source latitude relative to the ecliptic plane using the solar $B$ angle. There were 27 westward and 14 eastward Flong components and 16 eclipticward and 25 poleward Flat components among the 41 total events.

We do a series of comparisons to look for any significant correlations between the SEP event and $\mathrm{CH} F$ parameters. The 


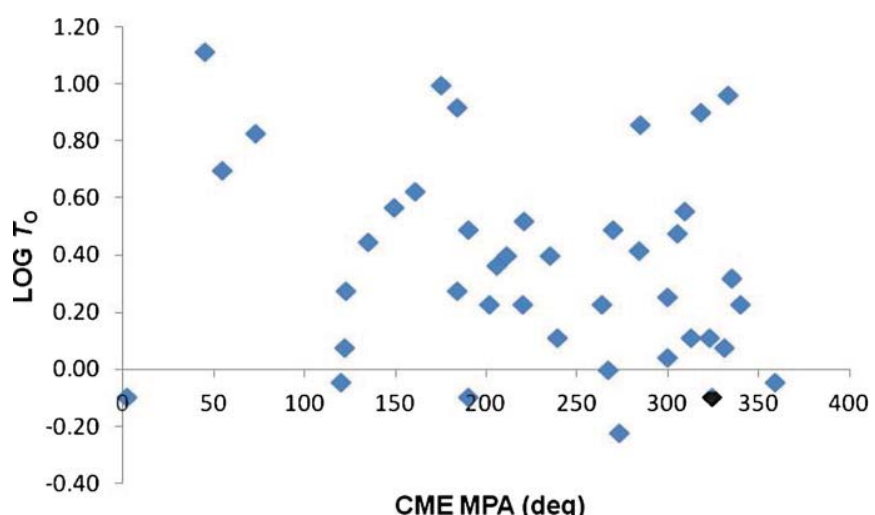

Figure 4. Logs of the SEP event onset times $T_{O}$ vs. the associated CME MPA directions. Most MPAs are in the western hemisphere $\left(180^{\circ}<\mathrm{MPA}<360^{\circ}\right)$, and there is a slight but not significant negative correlation of $T_{O}$ with MPA (Table 2), suggesting a marginally shorter $T_{O}$ for westward propagating and better connected CMEs.

(A color version of this figure is available in the online journal.)

Table 2

Correlation Coefficients between the SEP Events and $\mathrm{CH}$ or CME Parameters

\begin{tabular}{lrrrc}
\hline \hline SEP/CME (No. of Events) & Log Ipeak & $\log T_{O}$ & $\log T_{R}$ & $90 \% \mathrm{CC}$ \\
\hline Log F (41) & 0.24 & 0.17 & 0.11 & 0.26 \\
Flong (41) & 0.00 & 0.14 & 0.06 & 0.26 \\
Flat (41) & 0.09 & -0.21 & -0.06 & 0.26 \\
Log West Flong (27) & -0.07 & 0.25 & -0.10 & 0.32 \\
Log East Flong (14) & 0.10 & 0.22 & -0.00 & 0.46 \\
Log Equat Flat (16) & 0.27 & -0.28 & 0.05 & 0.43 \\
Log Pole Flat (25) & -0.03 & -0.35 & -0.22 & 0.34 \\
MPA (41) & 0.06 & -0.21 & -0.17 & 0.26 \\
\hline
\end{tabular}

correlation coefficients (CC) are presented in Table 2 along with the $\mathrm{CC}$ required for a modest $90 \%$ probability of correlation (Bevington \& Robinson 2003) in the last column. If we consider that the directions rather than the magnitude of $F$ would be important for ordering SEP events by producing better or worse magnetic connections of SEP sources, then any correlations of SEP parameters with $\log F$, given in the first line of Table 2, would not be expected. We compared the SEP parameters with linear values of Flong and Flat, which ranged over negative and positive values, and then separately with logs of the east, west, equatorward, and poleward components, respectively in rows four to seven of Table 2. The statistics are now diminishing, but the only hints of correlations seem to go in an unexpected direction, such as that $(\mathrm{CC}=-0.35,90 \%$ significance for 25 events) of the shorter onset times $T_{O}$ with increasing strengths of the poleward deflections of Flat or with decreasing strengths of the westward deflections. The primary result here is that we find no significant correlations between the SEP event and $\mathrm{CH}$ $F$ parameters, as shown in Table 2.

\subsubsection{The SEP Parameters versus the CME MPAs}

The analysis of the previous section was based on the two assumptions that the SEP event parameters might be dependent on the propagation direction of the CME and that the CME propagation would be deflected by the $\mathrm{CH}$ magnetic fields. The lack of any correlation between the $\mathrm{CH} F$ vectors and the SEP event parameters now raises two questions. First, do the SEP parameters depend on the CME propagation directions, and second, do the $\mathrm{CH}$ fields significantly deflect the CMEs? We can use our data set of 41 SEP events and CMEs to investigate both questions.

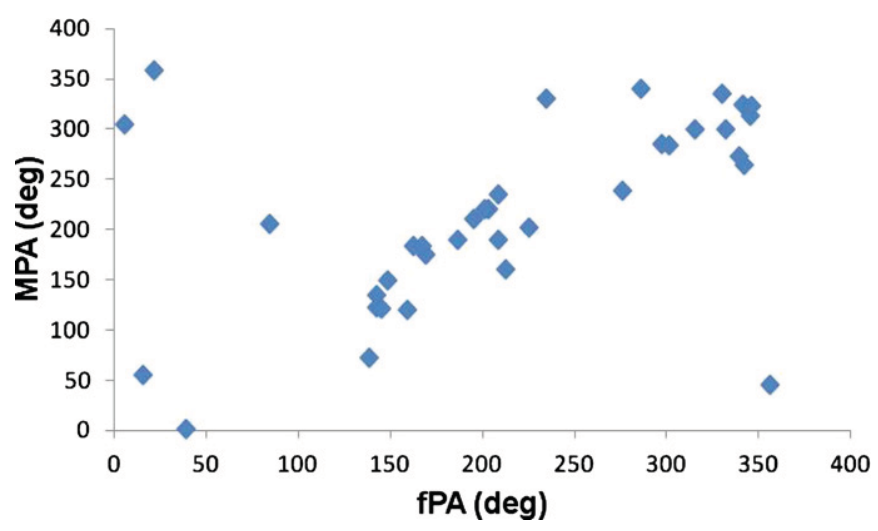

Figure 5. Source flare PA (fPA) correlates with the observed CME MPA at $\mathrm{CC}=0.47$ for the 36 events. A modified plot (see the text) yields a $\mathrm{CC}=0.91$, suggesting a radial propagation of the CME from the flare site.

(A color version of this figure is available in the online journal.)

We might expect that the SEP timescales are shortest and peak intensities highest for westward CME propagation directions and reversed for unfavorable eastward CME directions, resulting in extreme values at CME MPAs centered on $\sim 270^{\circ}$ and $\sim 90^{\circ}$, respectively. However, plotting each of the SEP event parameters against the CME MPAs, we find no significant correlations, as shown on the last line of Table 2. While the plot of $\log T_{0}$ against the CME MPA in Figure 4 is slightly suggestive of the expected shorter values at $\sim 270^{\circ}$, the characteristically broad scatter of $T_{0}$ across the MPA values indicates an independence of the SEP parameters on the directions of CME propagation from CM source regions.

\subsubsection{Significance of the Flare Locations and $F$ Vectors for CME MPAs}

The next question is how effectively the $\mathrm{CH}$ magnetic force $F$ moderates the CME MPAs. We first converted the heliographic latitude coordinates of the associated flare locations to ecliptic latitude coordinates by correcting for the solar $B$ angle and then calculated a solar PA relative to the sub-Earth point for each of the associated 36 flare sources lying outside a $15^{\circ}$ radius of the sub-Earth point. The source flare PA (fPA) correlates with the observed MPA at $\mathrm{CC}=0.47$, well above the $99 \%$ significant level, as shown in Figure 5. However, if we are mindful of the discontinuity of adjacent PAs from $0^{\circ}$ to $360^{\circ}$ and reorganize the plot by converting two fPA values in the upper left corner from $5^{\circ}$ and $21^{\circ}$ to $365^{\circ}$ and $381^{\circ}$, respectively, and a third value in the lower right of the plot from $356^{\circ}$ to $-4^{\circ}$, then the $\mathrm{CC}$ rises to 0.91 , well exceeding the $99.9 \%$ significance level. Thus the fPA is a reasonable guide to the MPA of the CME propagation, which suggests a generally radial propagation of the CMEs from their source regions independent of any $\mathrm{CH}$ effects.

Despite the excellent agreement of the CME MPAs with the fPAs in Figure 5, we can still compare the PAs of the $F$ vectors (FPAs) with the 41 observed MPAs to look for a correlation that would indicate CME deflections in the directions of the $F$ vectors. The plot of Figure 6 has a correlation of $\mathrm{CC}=0.36$, significant at the $98 \%$ significance level. There is more scatter in this plot than that of the MPA versus fPA of Figure 5, which suggests that the $\mathrm{CH}$ magnetic fields may play some role in deflecting CME propagation directions. 


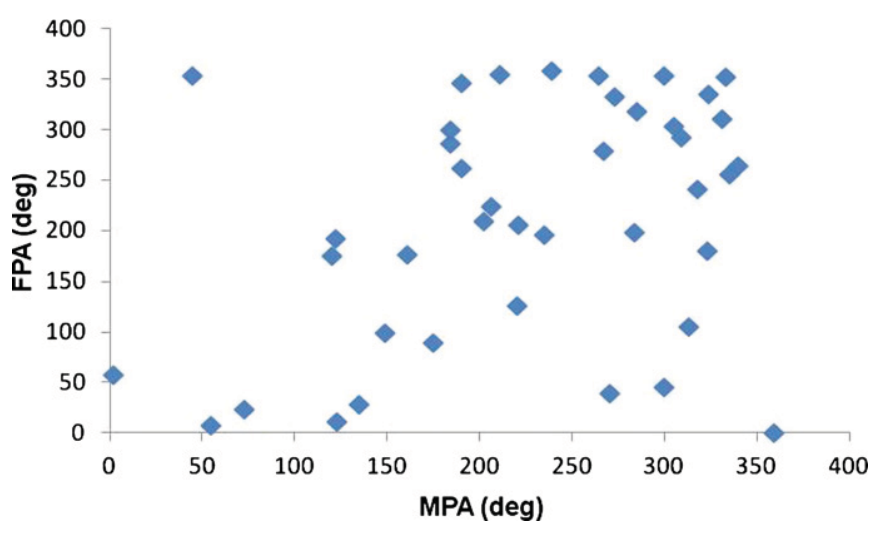

Figure 6. Comparison of the $41 \mathrm{CH}$ FPA values with the observed MPAs also shows a significant correlation consistent with an effect of the $\mathrm{CH}$ fields on the direction of CME propagation.

(A color version of this figure is available in the online journal.)

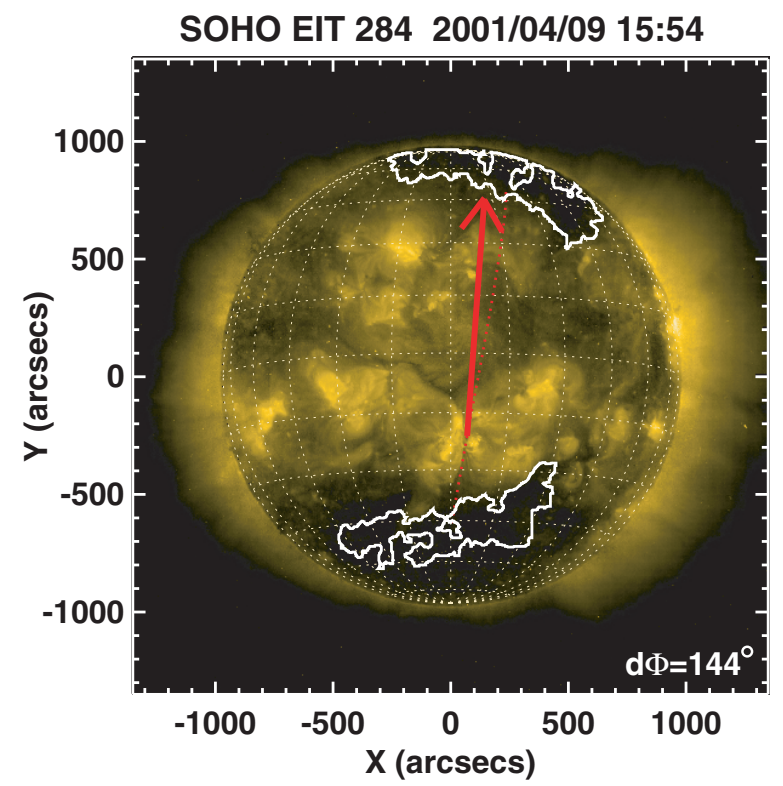

Figure 7. DPA may not be a useful parameter to judge the effect of $\mathrm{CH}$ fields on CMEs, as illustrated in the 2001 April 9 event in the same format as Figure 3. The flare source site $15^{\circ}$ south of the sub-Earth point implies a southward CME propagation, which may have been modified by the southern polar $\mathrm{CH}$ toward a lower latitude course, but not sufficient to change the MPA of $211^{\circ}$

(A color version of this figure is available in the online journal.)

\subsubsection{The DPA as a Further Test of CH Field Effectiveness}

Gopalswamy et al. (2009) calculated the difference between the MPA and the FPA, defined as the DPA $(\Delta \psi$ in Mohamed et al. 2012), a measure of how well the CME propagation angle correlated with that expected from an effective $\mathrm{CH} F$ vector. In their examination of shocks without observed driver ICMEs, they found a better agreement, i.e., smaller DPA values, for those CM CMEs not observed at 1 AU than for those that were observed. This result was consistent with the idea that CMEs not observed at $1 \mathrm{AU}$ were effectively deflected by the $\mathrm{CH}$ magnetic forces. However, the DPA may not always be a useful parameter to judge effects of $\mathrm{CH}$ fields on CMEs, as illustrated for the 2001 April 9 event shown in the EIT image of Figure 7. The DPA for that event is $144^{\circ}$, a large value resulting from an MPA of $211^{\circ}$ and an FPA of $355^{\circ}$. A second SEP event on the following day with the same source region had a similarly high DPA of $156^{\circ}$. Since the CME source region lies south of the sub-

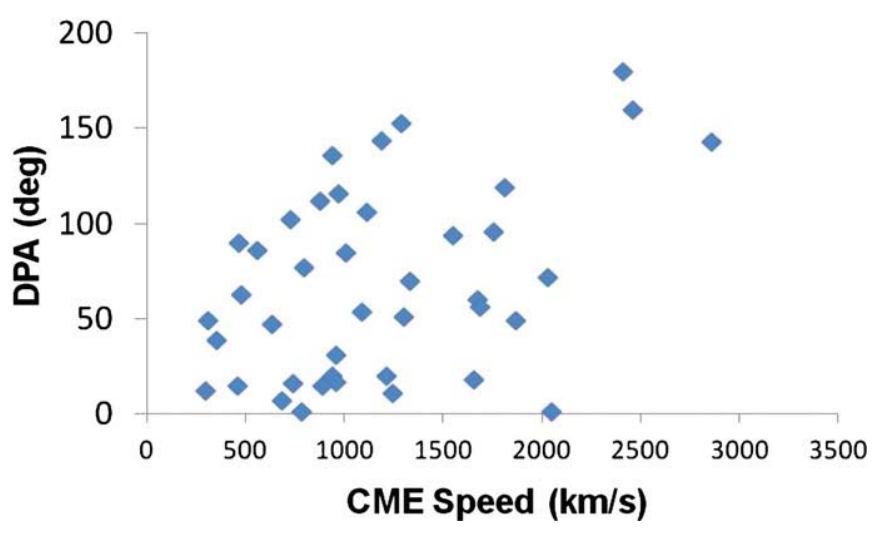

Figure 8. DPA vs. the CME speeds for the 41 SEP events. Larger DPA values indicate worse agreement between the CME propagation direction MPA and the $\mathrm{CH}$ force vector $F$ direction. This result, significant at the $90 \%$ level, supports the concept the $\mathrm{CH}$ deflection force is less effective for faster CMEs, presumed to have larger momenta than slower CMEs.

(A color version of this figure is available in the online journal.)

Earth point, we expect that the MPA should be southward, near $180^{\circ}$, as observed. The expected effect of the magnetic fields from the dominant southern $\mathrm{CH}$ should be to deflect the $\mathrm{CME}$ equatorward, but if that deflection occurred, it was not sufficient to change the resulting southward projection of the CME.

With this caveat of a projection effect in mind, we have compared DPA $=|\mathrm{MPA}-\mathrm{FPA}|$ with $\log F$ to see whether, as the $\mathrm{CH}$ magnetic force increases, the DPA becomes smaller, indicating better agreement with the expected deflection PA and resulting in a negative correlation between the two parameters. However, we instead calculate a small positive $\mathrm{CC}=0.15$, below the significance value of 0.26 , but opposite to the expected result for significant $\mathrm{CH}$ field effects on CMEs. A test for the projection effect is to look for a trend for the CME sources with angular separations farthest from the sub-Earth point to have the largest DPAs, as we discussed for the example of Figure 7. However, we find $\mathrm{CC}=0.02$ for DPA versus source separation, consistent with no effect of the $\mathrm{CH} F$ on the CME MPA.

Another approach is to ask whether the faster CMEs are less deflected than slower CMEs, with presumed smaller momenta, by the $\mathrm{CH}$ fields, as suggested by Wang et al. (2004) and Gui et al. (2011). For this purpose we compared the DPA with CME speed and found a $\mathrm{CC}=0.28$, a barely significant value at the $90 \%$ significance level, but clearly supportive of an effective $\mathrm{CH}$ force on CME propagation (Figure 8). Thus, the DPA correlation analyses give conflicting results, increasing with $F$, but decreasing with $\mathrm{CME}$ speed. If we give more weight to the latter result, then the DPA comparisons provide weak evidence for significant deflections of CMEs by the $\mathrm{CH}$ fields.

\section{SUMMARY AND DISCUSSION}

\subsection{Effects of CH Fields and CME Propagation on SEP Events}

The recent result that $\mathrm{CH}$ magnetic fields can significantly deflect shock-producing CMEs from near CM (Gopalswamy et al. 2009, 2010) raised the possibility that these deflections may have significant consequences for the SEP events produced by particle acceleration in the shocks driven by CMEs. We used a sample of $4120 \mathrm{MeV}$ SEP events with associated CMEs from CM locations to look statistically for systematic variations in SEP onset $T_{O}$ and rise $T_{R}$ times and peak intensities Ip resulting from $C M E$ deflections. An ad hoc force parameter $F$ connects the $\mathrm{CH}$ properties of magnetic-field intensity, area, and proximity to 
the expected CME deflection. The question is whether the broad range of SEP event peak intensities (Kahler 2001) and timescales (Kahler 2005) can be organized in any way by the use of the calculated $F$ vector. We first sought a direct connection between SEP parameters and the magnitude or direction of the $F$ vectors, which were resolved into equatorward/poleward and east/west components. We found no statistically significant correlations between these two sets of parameters, as indicated in Table 2.

The independence of SEP event parameters from expected CME deflections led us to ask whether the SEP parameters depend on the CME propagation directions as defined by their MPAs. SEP event onset and rise times are dependent on the longitudes of the solar source regions (Kahler 2005), but with a selection of source regions from a longitudinal band only $40^{\circ}$ wide, we might expect that their associated CME propagation directions would provide some of the longitudinal organization lost by confining our source regions to the $40^{\circ}$ wide band. That this is not the case is obvious from Figure 4 in which we find no significant difference between eastward (MPA $\sim 90^{\circ}$ ) and westward $\left(\mathrm{MPA} \sim 270^{\circ}\right) \mathrm{CMEs}$ for $T_{O}$. The implication here is that even if $\mathrm{CH}$ fields are deflecting CMEs, those deflections are not relevant to the SEP events produced by the CME-driven shocks.

\subsection{CME Propagation Directions and CH Fields}

We also asked whether we find evidence, independent of the $\mathrm{SEP}$ events, for an impact of the $\mathrm{CH}$ magnetic fields in deflecting CMEs. Comparing first the CME propagation directions expected only on the basis of the associated fPAs, we found the good agreement shown in Figure 5. While this result could suggest an essentially undeflected radial propagation for those CM CMEs, we also found a modest but significant correlation between the FPA and MPA (Figure 6), consistent with some $\mathrm{CH}$ effect in deflecting the CME propagation direction. From their proximity to the Sun center, we can expect substantial scatter in the MPAs of the CMEs of this study, which may contribute to the scatter of Figure 6. The MPA provides us with only an azimuthal direction projected on the plane of the sky, so significant CME deflections of propagation from radial can go undetected, as illustrated in Figure 7 showing an FPA almost opposite in direction from the observed MPA. This could explain why we find a weak increase of DPA with increasing magnitude of $F$, a result contrary to the expectation that stronger $\mathrm{CH}$ fields $F$ should be more effective in deflecting CMEs. STEREO/SECCHI observations (e.g., Liu et al. 2010) will be required to determine the actual deflections from radial propagation that might result from $\mathrm{CH}$ fields.

An alternative and complementary approach to look for evidence of CME deflections is to compare latitudinal deviations of limb CMEs with their calculated $\mathrm{CH}$ latitudinal $F$ vectors. This approach has obvious limitations in determining appropriate $\mathrm{CH}$ properties, but avoids the projection limitations of this study indicated in Figure 7. The results of such a study, shown in Figure 6 of Cremades et al. (2006), indicate a good correlation between CME deviations and $F$ vectors.

As a second test of the effectiveness of $\mathrm{CH}$ fields in deflecting CMEs, we followed the assertions of Wang et al. (2004) and Gui et al. (2011) that slower CMEs should be more susceptible than faster CMEs to the deflecting forces of $\mathrm{CH}$ fields. Our comparison of DPA, the angular difference between MPA and FPA, with CME speed shows good agreement with that idea. The results shown in Figures 6 and 8 further support the concept of CME deflections by CH fields (Cremades et al. 2006; Gopalswamy et al. 2009).

\subsection{Implications and Limitations of the Study}

A fraction of the variability of SEP event timescales is dependent on solar source longitude (Kahler 2005), but we find no evidence that within a confined longitude range SEP event parameters depend on the CME propagation directions defined by their MPAs. We had expected from Figure 1 that CME propagation directions should be at least a contributing factor for the access of SEPs to Earth-connected interplanetary field lines and would be manifested in the statistical distributions of the SEP event timescales and perhaps peak intensities. In an earlier study Kahler et al. (2000) found that SEP peak intensities were independent of CME latitudes for a set of $17 \mathrm{SEP}$ events limited to an SMMC/P CME speed range of $650-850 \mathrm{~km} \mathrm{~s}^{-1}$ and source longitudes of $>\mathrm{W} 20^{\circ}$. Our work with $\mathrm{CM}$ sources allows us to examine a full range of CME MPAs and extend the comparisons to include SEP event timescales. The lack of any SEP event dependence on the MPAs of these CMEs is consistent with SEP production in CME-driven shocks much broader than those suggested in the schematic of Figure 1. SEP events (Kahler $\&$ Reames 2003) and type II radio shock bursts (Gopalswamy et al. 2001) are associated only with CME widths $\gtrsim 60^{\circ}$, but the inferred angular extents of shocks at $1 \mathrm{AU}$ can reach $\gtrsim 100^{\circ}$ (Gopalswamy et al. 2001; de Lucas et al. 2011), and SEP events have been observed $\sim 180^{\circ}$ from their source regions (Cliver et al. 2005).

Figures 6 and 8 show correlations supporting the idea of $\mathrm{CH}$ deflections of CMEs, but one possible bias is that $\mathrm{CHs}$ are often located between the CME source active regions and Sun center such that the $F$ vectors coincidently align with the CME MPAs determined primarily by the CME source locations. This could then give rise to the apparent correlation of FPAs with MPAs of Figure 6 . We think this is unlikely since $F$ is typically calculated from multiple $\mathrm{CHs}$. In using the associated flare locations as the CME sources we have ignored offsets of CME sources from active region centers (Wang \& Zhang 2007) and CME deflections from nearby sunspot fields (Sterling et al. 2011). Our study was limited to SEP events with sources within $20^{\circ}$ of $\mathrm{CM}$ and was based on an ad hoc force vector $F$ calculated from the properties of all CHs observed on the Sun at the time of each SEP event. We can not rule out the possibility that SEP events with origins at other longitude regions may in fact show some $\mathrm{CH}$ dependence, but our result implies that effects of $\mathrm{CHs}$ are not of importance for forecasting properties of SEP events. Finally, we point out that this is only a coronal study, which does not include a consideration of the extension of the $\mathrm{CH}$ environment to the fast SW stream regions at 1 AU. Effects of CHs may still be important for SEPs, but only in the interplanetary medium where the magnetic connectivity of the observer to particular solar source regions, and $\mathrm{CHs}$ in particular, may play a significant role. A complementary study to compare these SEP events with interplanetary fields and their solar footpoints will be done.

S. Kahler was funded by AFOSR Task 2301RDZ4. N. Gopalswamy and S. Akiyama were supported by NASA's LWS TR\&T program. CME data were taken from the CDAW LASCO catalog. This CME catalog is generated and maintained at the CDAW Data Center by NASA and The Catholic University of America in cooperation with the Naval Research Laboratory. $\mathrm{SOHO}$ is a project of international cooperation between ESA and NASA. 


\section{REFERENCES}

Bevington, P. R., \& Robinson, D. K. 2003, Data Reduction and Error Analysis (Boston, MA: McGraw-Hill)

Bothmer, V., \& Zhukov, A. 2007, in Space Weather Physics and Effects, ed. V. Bothmer \& I. A. Daglis (Berlin: Springer), 31

Byrne, J. P., Maloney, S. A., McAteer, J., Refojo, J. M., \& Gallagher, P. T. 2010, Nat. Commun., 74

Cliver, E. W. 2009, in IAU Proc. 257, Universal Heliospheric Processes, ed. N. Gopalswamy \& D. F. Webb (Cambridge: Cambridge Univ. Press), 401

Cliver, E. W., et al. 2005, Proc. 29th Int. Cosmic Ray Conf., 1, 121

Cremades, H., Bothmer, V., \& Tripathi, D. 2006, Adv. Space Res., 38, 461

Crosby, N. B. 2007, in Space Weather Physics and Effects, ed. V. Bothmer \& I. A. Daglis (Berlin: Springer), 131

de Lucas, A., Schwenn, R., dal Lago, A., Marsch, E., \& Clúa de Gonzalez, A. L. 2011, J. Atmos. Sol.-Terr. Phys., 73, 1281

Gopalswamy, N., Makela, P., Xie, H., Akiyama, S., \& Yashiro, S. 2009, J Geophys. Res., 114, A00A22

Gopalswamy, N., Makela, P., Xie, H., Akiyama, S., \& Yashiro, S. 2010, in AIP Conf. Proc. 1216, Twelfth Int. Solar Wind Conf., ed. M. Maksimovic et al. (Melville, NY: AIP), 452

Gopalswamy, N., \& Thompson, B. J. 2000, J. Atmos. Terr. Phys., 62, 1457

Gopalswamy, N., Yashiro, S., Kaiser, M. L., Howard, R. A., \& Bougeret, J.-L. 2001, J. Geophys. Res., 106, 29219

Gopalswamy, N., Yashiro, S., Kaiser, M. L., et al. 2002, ApJ, 572, L103

Gopalswamy, N., Yashiro, S., Krucker, S., Stenborg, G., \& Howard, R. A. 2004, J. Geophys. Res., 109, A12105

Gui, B., Shen, C., Wang, Y., et al. 2011, Sol. Phys., 271, 111

Guetersloh, S., \& Zapp, N. 2010, in Heliophysics: Space Storms and Radiation: Causes and Effects, ed. C. J. Schrijver \& G. L. Siscoe (Cambridge: Cambridge Univ. Press), 359

Kahler, S., \& Webb, D. 2010, in AIP Conf. Proc. 1216, Twelfth Int. Solar Wind Conf., ed. M. Maksimovic et al. (Melville, NY: AIP), 408

Kahler, S. W. 2001, J. Geophys. Res., 106, 20947

Kahler, S. W. 2004, ApJ, 603, 330

Kahler, S. W. 2005, ApJ, 628, 1014
Kahler, S. W., Cliver, E. W., \& Ling, A. G. 2007, J. Atmos. Sol.-Terr. Phys., 69, 43

Kahler, S. W., \& Reames, D. V. 2003, ApJ, 584, 1063

Kahler, S. W., Reames, D. V., \& Burkepile, J. T. 2000, in ASP Conf. Ser. 206, High Energy Solar Physics: Anticipating HESSI, ed. R. Ramaty \& N Mandzhavidze (San Francisco, CA: ASP), 468

Kahler, S. W., Tylka, A. J., \& Reames, D. V. 2009, ApJ, 701, 561

Kahler, S. W., \& Vourlidas, A. 2005, J. Geophys. Res., 110, A12S01

Kilpua, E. K. J., Pomoell, J., Vourlidas, A., et al. 2009, Ann. Geophys., 27, 4491

Klecker, B., Mobius, E., \& Popecki, M. A. 2007, Space Sci. Rev., 130, 273

Kunches, J. M., \& Zwickl, R. D. 1999, Rad. Meas., 30, 281

Liu, Y., Thernisien, A., Luhmann, J. G., et al. 2010, ApJ, 722, 1762

Lugaz, N., Downs, C., Shibata, K., et al. 2011, ApJ, 738, 127

Malandraki, O. E., Marsden, R. G., Lario, D., et al. 2009, ApJ, 704, 469

McKibben, R. B., Connell, J. J., Lopate, C., et al. 2003, Ann. Geophys., 21, 1217

Mohamed, A. A., Gopalswamy, N., Yashiro, S., et al. 2012, J. Geophys. Res., 117, A01103

Panasenco, O., Martin, S., Joshi, A. D., \& Srivastava, N. 2011, J. Atmos. Sol.-Terr. Phys., 73, 1129

Panasenco, O., Martin, S., Velli, M., \& Vourlidas, A. 2012, Sol. Phys., in press

Reames, D. V. 1999, Space Sci. Rev., 90, 413

Reames, D. V., Kahler, S. W., \& Ng, C. K. 1997, ApJ, 491, 414

Sanderson, T. R. 2004, in The Sun and Heliosphere as an Integrated System, ed. G. Poletto \& S. T. Suess (Dordrecht: Kluwer), 113

Shen, C., Wang, Y., Gui, B., Ye, P., \& Wang, S. 2011, Sol. Phys., 269, 389

Shen, C., Wang, Y., Ye, P., \& Wang, S. 2006, ApJ, 639, 510

Shen, C., Yao, J., Wang, Y.-M., et al. 2010, Res. Astron. Astrophys., 10, 1049

Sterling, A. C., Moore, R. L., \& Harra, L. K. 2011, ApJ, 743, 63

Tylka, A. J., \& Lee, M. A. 2006, ApJ, 646, 1319

Wang, Y.-M. 2009, Space Sci. Rev., 144, 383

Wang, Y., Chen, C., Gui, B., et al. 2011, J. Geophys. Res., 116, A04104

Wang, Y., Shen, C., Wang, S., \& Ye, P. 2004, Sol. Phys., 222, 329

Wang, Y., \& Zhang, J. 2007, ApJ, 665, 1428

Zuccarello, F. P., Bemporad, A., Jacobs, C., et al. 2012, ApJ, 744, 66 